\title{
A study of $R_{V}$ in Galactic $O$ stars from the 2 MASS catalogue $^{\star, \star \star}$
}

\author{
P. Patriarchi ${ }^{1}$, L. Morbidelli ${ }^{1}$, and M. Perinotto ${ }^{2}$ \\ 1 IRA/CNR, Largo E. Fermi 5, 50125 Firenze, Italy \\ ${ }^{2}$ Dipartimento di Astronomia e Scienza dello Spazio, Universitá di Firenze, Largo E. Fermi 2, 50125 Firenze, Italy
}

Received 17 October 2002 / Accepted 24 July 2003

\begin{abstract}
We present new measurements of the interstellar reddening parameter $R_{V}=A_{V} / E(B-V)$ towards $185 \mathrm{O}$ stars, using $J, H, K_{\mathrm{s}}$ photometry from the 2MASS project. The results are combined with data from the literature of 95 stars where $R_{V}$ has been derived with the same technique, 22 of which in common with our present sample from the 2MASS project catalogue. The average $R_{V}$ from these $258 \mathrm{O}$ stars is of $3.19 \pm 0.50$. All objects whose $R_{V}$ departs from this value by more than $2 \sigma$ have been recognized. Ten objects have $R_{V}$ higher than this value and two lower. It is found that anomalous $R_{V}$ can scarcely be associated with anomalies in the general interstellar medium, e.g. with different behaviour in different spiral arms. They are clearly linked to local cloud effect. In the Cygnus region $R_{V}$ values follow the behaviour of the general interstellar medium, while in the Carina arm, in spite of the relatively larger distance, local cloud effects prevail. An explanation for this is suggested.

The relatively few stars of our sample whose Hipparcos parallaxes are reliable, are found to have distances systematically smaller than the distances derived by the spectroscopic parallaxes. We argue that this effect is consistent with the recently claimed discovery of grey extinction towards OB stars.
\end{abstract}

Key words. ISM: dust, extinction

\section{Introduction}

$R_{V}$ is the single basic parameter which characterizes the interstellar extinction from the near-infrared to the far-UV spectral region. The absolute extinction $A_{V}$, from which $R_{V}$ is derived, is best determined by optical and near-infrared photometry (Cardelli et al. 1989, CCM89). They found that the extinction $A_{\lambda} / A_{V}$ in the range $0.125-3.5 \mu \mathrm{m}$ depends in practice only on $R_{V}$. The same authors also showed that the absolute extinction $A_{V}$, and consequently $R_{V}$, are best determined from optical and near-infrared photometry. $\mathrm{O}$ stars are particularly suited to investigate the behaviour of the interstellar extinction to large distancies in the Galaxy.

Send offprint requests to: $\mathrm{P}$. Patriarchi,

e-mail: pat@arcetri.astro.it

* This publication makes use of data products from the Two Micron All Sky Survey (2MASS), which is a joint project of the University of Massachusetts and the Infrared Processing and Analysis Center/California Institute of Technology, funded by the National Aeronautics and Space Administration and the National Science Foundation.

$\star \star$ Tables 1 and 2 are only available in electronic form at the CDS via anonymous ftp to cdsarc.u-strasbg.fr $(130.79 .128 .5)$ or via http://cdsweb.u-strasbg.fr/cgi-bin/qcat?J/A+A/410/905
So far the $R_{V}$ parameter has been determined directly towards 95 O stars in the Galaxy (Patriarchi et al. 2001, Paper I). It is clearly important to extend this type of information to as many lines of sight as possible, in order to reach the best knowledge on the nature of the interstellar dust across the Galaxy.

We considered that the 2MASS (Two Micron All Sky Survey) catalogue can contribute significantly to this, containing $J, H, K_{\mathrm{s}}$ photometry of many stars. This allowed us to obtain a new sample of $185 \mathrm{O}$ stars where we could precisely determine the $R_{V}$ quantity. Adding the previously examined stars (see Paper I), the total sample of O stars with well measured $R_{V}$ is now 258 stars.

In Sect. 2 the characteristics of the 2MASS catalogue are recalled. Section 3 contains the determinations of $A_{V}$ and $R_{V}$ from the 2MASS catalogue. In Sect. 4 a discussion follows.

\section{The 2MASS catalogue}

The Two Micron All Sky Survey (2MASS) project has been designed to close the gap between the current technical capability in the near-infrared and the actual knowledge of the near-infrared sky. In addition to providing a context for the interpretation of results obtained at infrared and other wavelengths, 2MASS should provide 
direct answers to immediate questions on the large-scale structure of the Milky Way and the Local Universe (see http://pegasus.phast. umass.edu/frame.html). The optimal use of the next generation of infrared space missions, as well as powerful ground-based facilities, require a new census of sources with vastly improved sensitivity and astrometric accuracy over what was previously available.

To achieve these goals 2MASS has uniformly scanned the entire sky in three near-infrared bands to detect and characterize point sources brighter than about $1 \mathrm{mJy}$ in each band, with signal-to-noise ratio greater than 10, using a pixel size of 2.0 arcsec. This made it possible to achieve an 50000-fold improvement in sensitivity relative to earlier surveys, e.g. the "Two Micron Sky Survey" (Neugebauer \& Leighton 1969).

2MASS has used two highly-automated 1.3-m telescopes, one at Mt. Hopkins (USA) and one at CTIO, Chile. Each telescope was equipped with a three-channel camera, each channel consisting of a $256 \times 256$ array of $\mathrm{HgCdTe}$ detectors, capable of observing the sky simultaneously at $J(1.25 \mu \mathrm{m}), H(1.65 \mu \mathrm{m})$, and $K_{\mathrm{s}}(2.17 \mu \mathrm{m})$.

At the moment of writing this paper, two point-source catalogues have been released (the First and the Second Incremental Data Releases) covering more than half of the sky, while the remaining part of the sky has already been observed. Detailed information on the 2 MASS project can be found at the website cited above.

\section{Determination of $A_{V}$ and $\boldsymbol{R}_{V}$}

We have searched the 2MASS point-source catalogue for the presence of the O stars listed in the Garmany et al. (1982) Catalogue of galactic $\mathrm{O}$ stars. Only $\mathrm{O}$ stars having reliable $J$, $H, K_{\mathrm{s}}$ photometry have been considered. This resulted in 185 stars. From their spectral type, derived from the Garmany et al. catalogue, we have adopted $(B-V)_{0},(J-V)_{0},(H-V)_{0}$, $(K-V)_{0}$ from Wegner (1994), while $V$ and $(B-V)$ are from SIMBAD $U B V$ measurements. We have examined our new sample of 185 stars for membership in associations using again Garmany et al. (1982). Since Wegner's intrinsic colours are given in the SAAO system, they have been transformed to the 2MASS system according to the conversion formula given in the Explanatory Supplement to the 2MASS Second Incremental Data Release (Cutri et al. 2001). The photometric data of the considered 185 stars are reported in Table 1 together with their photometric errors, as given in the 2MASS catalogues.

The quantity $A_{V}$ was then determined from the $J, H, K_{\mathrm{s}}$ and $V$ photometry using the method developed by CCM89, who assumed that the shape of the IR extinction law is invariant, an assertion confirmed by Fitzpatrick (1999). This method consists in deriving $A_{V}$ from a least square fitting of the relationship

$E(\lambda-V)=A_{V}\left[R_{\mathrm{L}}(\lambda)-1\right]$

where $\lambda=J, H, K_{\mathrm{s}}$ and $R_{\mathrm{L}}$ is the extinction curve $A_{\lambda} / A_{V}$ by Rieke $\&$ Lebofsky (1985). From the derived $A_{V}$ and the known $E(B-V)$ we obtain $R_{V}$. Since the fitting expression (1) is a homogeneous equation, the uncertainty of each $A_{V}$ has

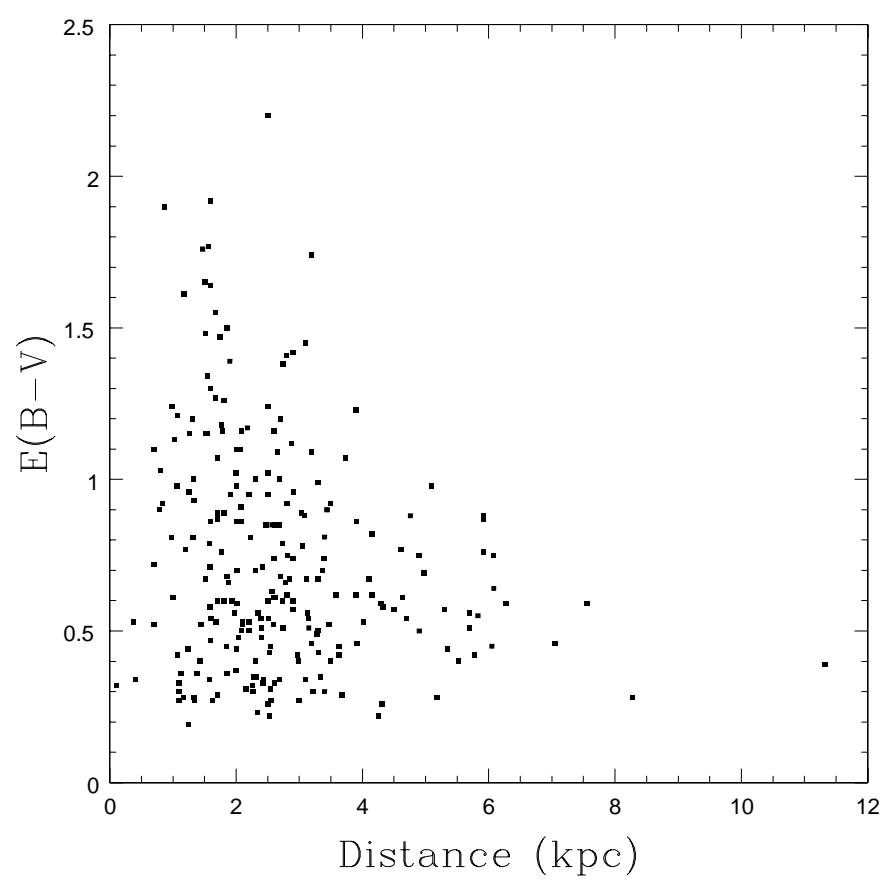

Fig. 1. $E(B-V)$ vs. distance for the total sample of galactic $\mathrm{O}$ stars with well determined $R_{V}$.

been calculated considering that we have $N-1$ degrees of freedom ( $N$ is the number of the photometric bands used). From the mentioned uncertainty of $A_{V}$ an estimate of the accuracy of $R_{V}$ has been derived. We report in Table 2 the full set of relevant quantities. From $A_{V}$ in Table 2 and $V$ in Table 1 we have derived the distances making use of the calibration $M_{V}$ vs. spectral type from Panagia (1973).

\section{Discussion}

\subsection{General interstellar medium vs. local clouds}

We first examine the behaviour of $E(B-V)$ and $A_{V}$ vs. distance. They are displayed for the total sample of our stars, including those studied in Paper I, in Figs. 1 and 2 for $E(B-V)$ and $A_{V}$ respectively. The two diagrams are obviously similar because the ratio $A_{V} / E(B-V)$ has in general an average value close to 3.1. The diagrams illustrate that distant stars, as expected, are seen only if they have low reddening.

In Fig. 3 we show $R_{V}$ vs. $E(B-V)$, again for all stars. Only at low reddening do we see a large spread of $R_{V}$ values. According to the general view larger $R_{V}$ are contributed to by relatively larger dust grains, while lower $R_{V}$ should be significantly produced by grain of smaller sizes. The diagram could be simply understood in the following terms. At low distances from the Sun the contribution of the general interstellar medium to the observed $R_{V}$ is unable to cancel out the effect of local clouds, which are known to frequently deviate from the average $R_{V}$ value of the interstellar medium. This is not true for larger distances where the general interstellar medium prevails over the effect of local clouds in the line of sight. We find that the above behaviour is more evident in this paper than in the past, because of the larger number of stars here studied homogeneously. 


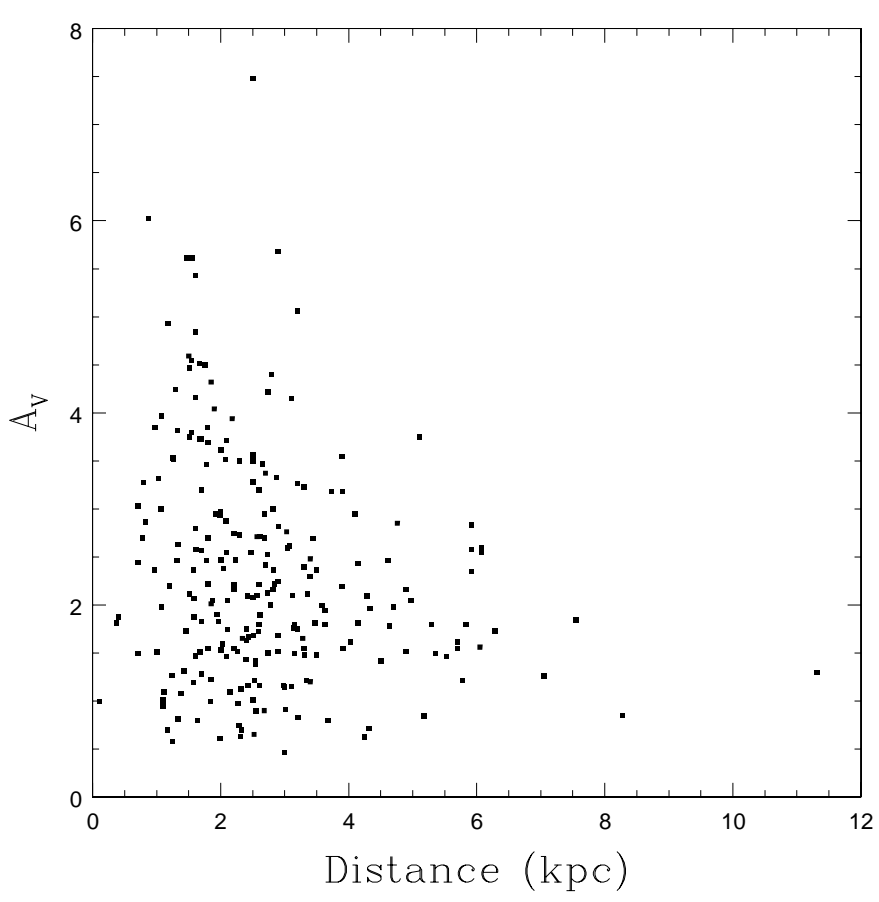

Fig. 2. $A_{V}$ vs. distance for the total sample.

The lines of sight with "anomalous" $R_{V}$ are marked in Table 2 with "s" and "l" for small and large $R_{V}$, respectively. Interesting enough, there are only two anomalous directions with small $R_{V}$ (and both stars are also peculiar), while there are eight with large $R_{V}$. This can be understood considering that we have examined lines of sight in front of $\mathrm{O}$ stars, which are more easily found in a $\mathrm{H}$ II environment, where $R_{V}$ is larger. It is interesting to note that five out of the eight " $\mathrm{l}$ " stars are emission-line stars, a number that greatly exceeds the proportion of the emission-line stars in the sample of 185 stars.

It is interesting to compare our derived $R_{V}$ values with those deduced from the equations A3-A5 of Fitzpatrick (1999) expressing $R_{V}$ as a function of the measured color excesses $(V-J),(V-H)$, and $(V-K)$. This is particularly useful to look for some "anomalous" differences (implying something wrong in our derived $R_{V}$ ). It is also a check of the correctness of the Fitzpatrick's expressions, since we are comparing them with our $R_{V}$ values derived observationally. The histograms of the relative differences $\left[R_{V}\right.$ (this paper) $R_{V}$ (Fitzpatrick)] $/ R_{V}$ (this paper) for each filter $J, H, K$ are shown in Fig. 4. No "anomaly" is visible, apart from small opposite biases of the histograms of the $J$ and $K$ filters with respect to the $H$ filter, whose histogram is centered around zero.

\subsection{Spectroscopic parallaxes vs. Hipparcos parallaxes}

Generally speaking the Hipparcos parallaxes of our sample of $\mathrm{O}$ stars may not be compared to our distance estimates because Hipparcos parallaxes are reliable only up to few hundred parsecs, while our stars are in general more distant. Anyway 8 stars have a relative error $\sigma_{\pi} / \pi \leq 0.5$, where $\pi$ is the Hipparcos parallax. Even comparing the distances derived by

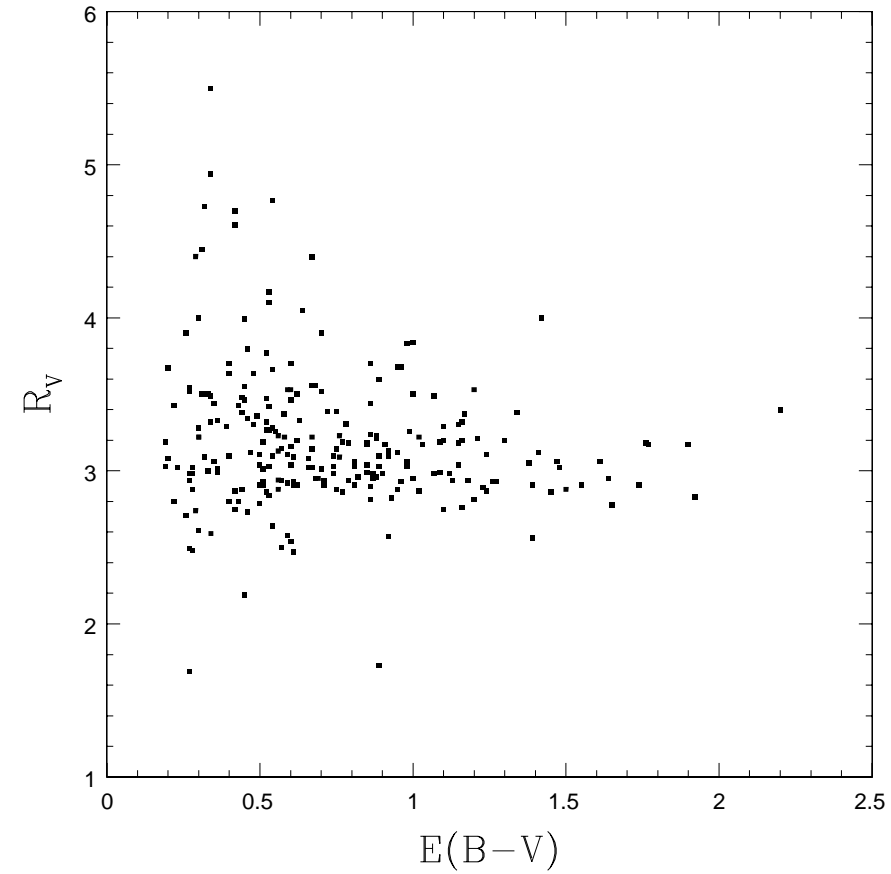

Fig. 3. $R_{V}$ vs. $E(B-V)$ for the total sample.

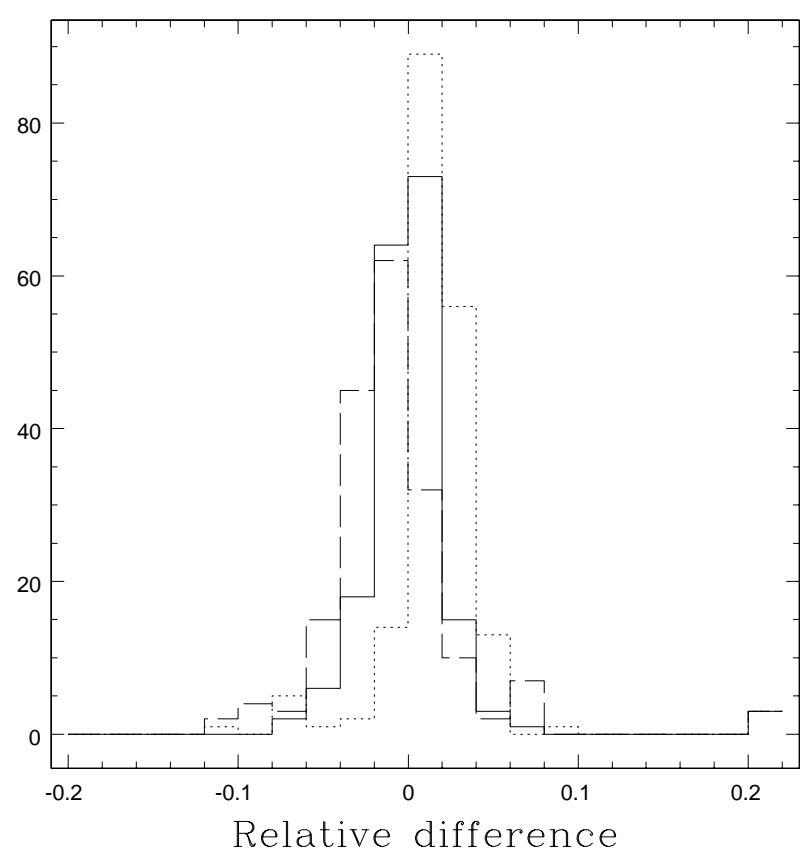

Fig. 4. Histogram of $\left[R_{V}\right.$ (this paper) $-R_{V}$ (Fitzpatrick) $] / R_{V}$ (this paper) for the $J$ (dash), $H$ (full), $K$ (dot) filters.

parallaxes equal to $\left(\pi-\sigma_{\pi}\right)$ to our distance estimates, the relative difference becomes $\approx-50 \% \pm 20 \%$, so our estimates seem systematically too large.

A recent paper of Skórzyński et al. (2003) claims the presence of a grey non-selective, i.e. not proportional to $E(B-V)$, extinction, at least in the direction of $O B$ stars. This kind of extinction does not influence colour excesses, nor, consequently, $A_{V}$ and $R_{V}$, so distances inferred by spectroscopic parallaxes could be systematically larger. Indeed the above 


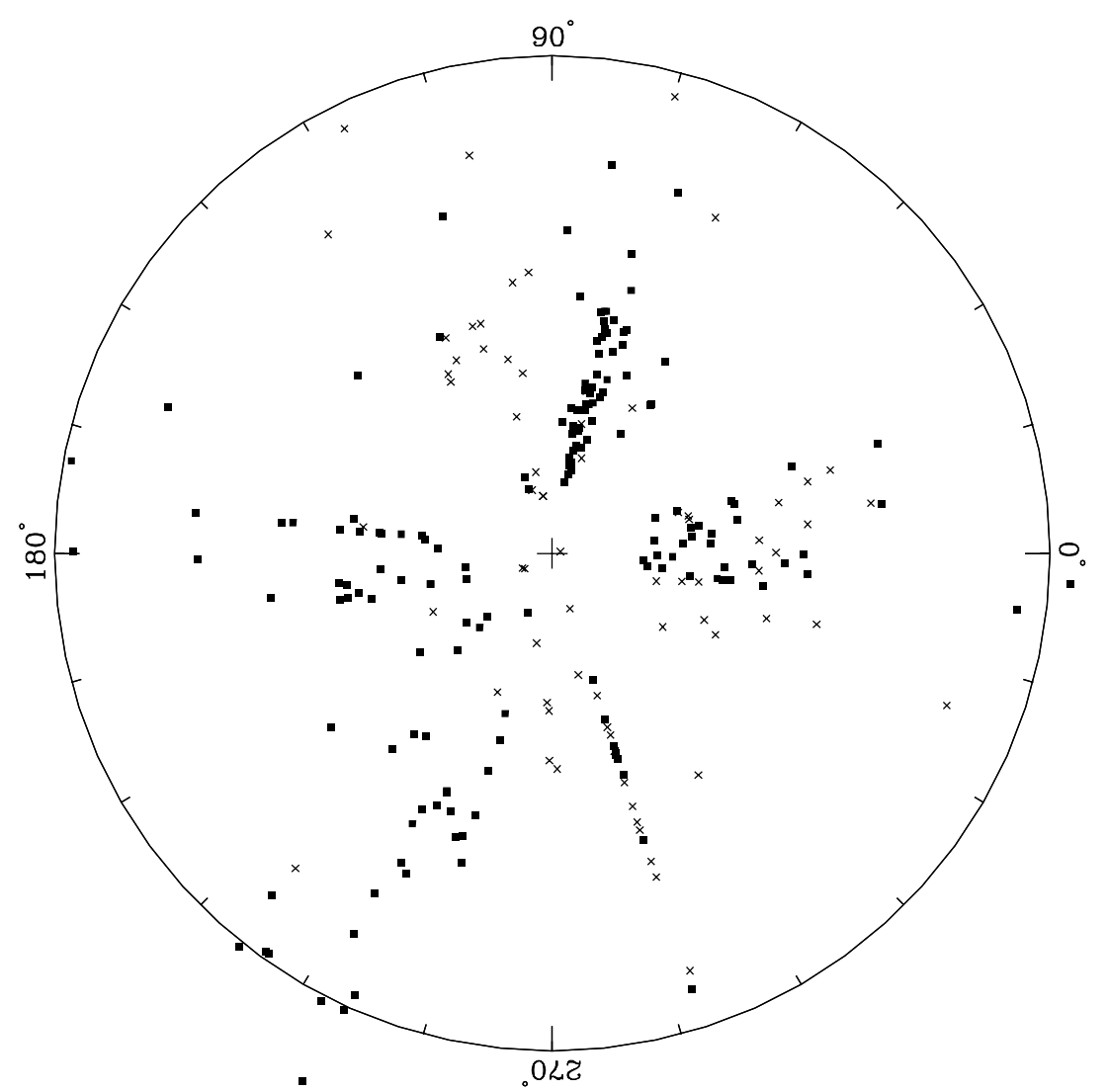

Fig. 5. All the $\mathrm{O}$ type stars with $R_{V}$ so far measured photometrically are plotted in the galactic plane centered on the Sun. Full squares denote the 2MASS stars, while crosses refer to $\mathrm{O}$ stars with $R_{V}$ from the literature. The circle has a radius of $6 \mathrm{kpc}$. Most stars belong to OB associations (see Table 2).

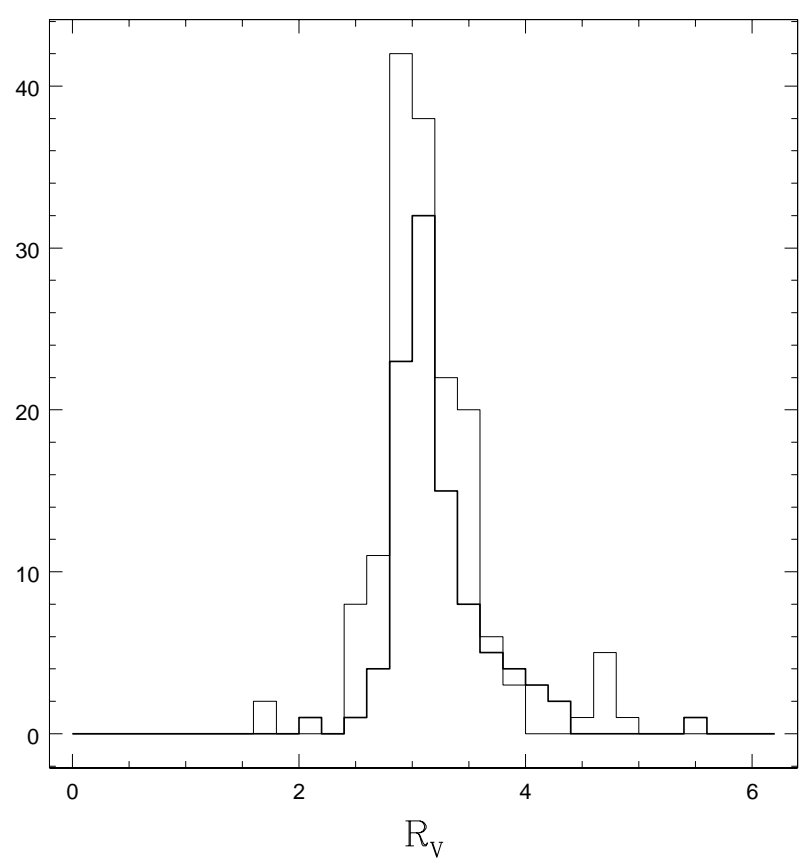

Fig. 6. Histograms of the stars belonging to clusters or associations (thick line) and of field stars (thin line). LSS stars of Paper I are not included (bin $=0.2$ ).

mentioned 8 objects with regular $E(B-V)$ for O stars, ranging from 0.23 to 0.68 , have Hipparcos distances of from $0.13 \mathrm{kpc}$ to $0.75 \mathrm{kpc}$, while spectroscopic distances range from $1.08 \mathrm{kpc}$ to $4.29 \mathrm{kpc}$. Let us consider, for example, the most extreme case, LS V+30 31. Its spectral type is O9 V corresponding to an absolute $V$ magnitude of -4.30 . Since it has an apparent $V$ magnitude of 10.95 for a Hipparcos distance of $0.13 \mathrm{kpc}$ and a "modest" $E(B-V)=0.59$, the grey extinction effect seems really present. Of course this topic deserves further study.

\subsection{Carina vs. Cygnus}

We now consider the behaviour of the total sample of the studied stars in the context of their location in the Galaxy. In Fig. 5 the stars are shown projected onto the galactic plane. The zones pertaining to the Sagittarius (Sgr OB1-7), Cygnus (Cyg OB1-9), Auriga (Aur OB1-2; Gem OB1), Puppis (CMa OB1; Pup OB1), and Carina (Trumpler 16, Collinder 228) associations are visible around the following galactic longitudes: $0,75,180,235,285$, respectively.

It is interesting to examine whether there are differences in the $R_{V}$ distribution between stars belonging to associations and young clusters on one side, and those considered to be field stars. This is done in Fig. 6. No significant differences between the two distributions are seen; the average $R_{V}$ are 3.24 and 3.15 for stars in groups and in the field respectively. We emphasize that the LSS stars of Paper I have not been included in these two samples, considering that owing to their faintness, their membership of clusters has not been investigated yet. The average $R_{V}$ value of the whole sample (LSS included) amounts to $3.19 \pm 0.50$. 


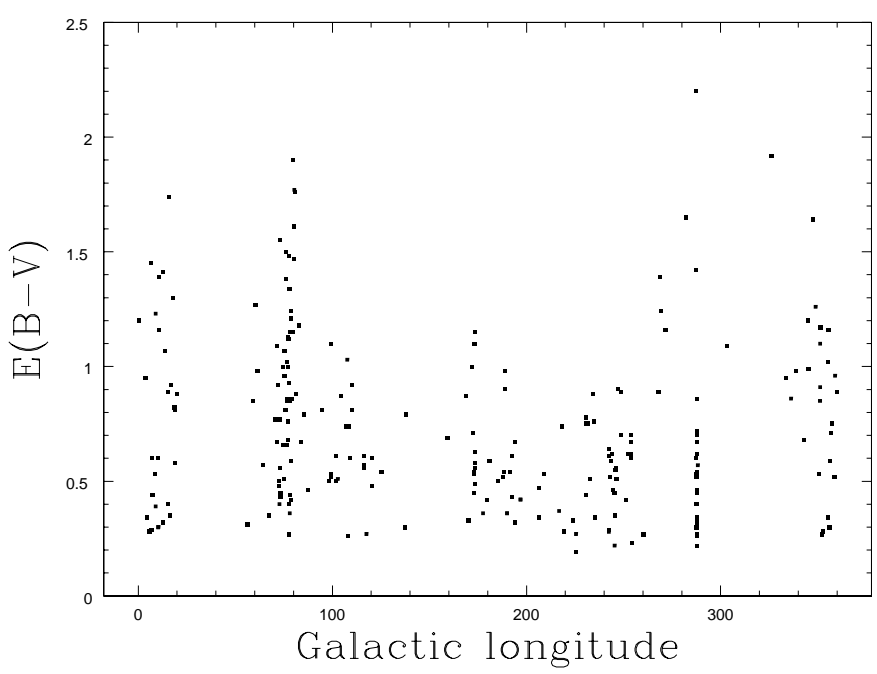

Fig. 7. $E(B-V)$ vs. galactic longitude for the total sample. Longitudes of Cygnus and Carina are 75 and 287, respectively.

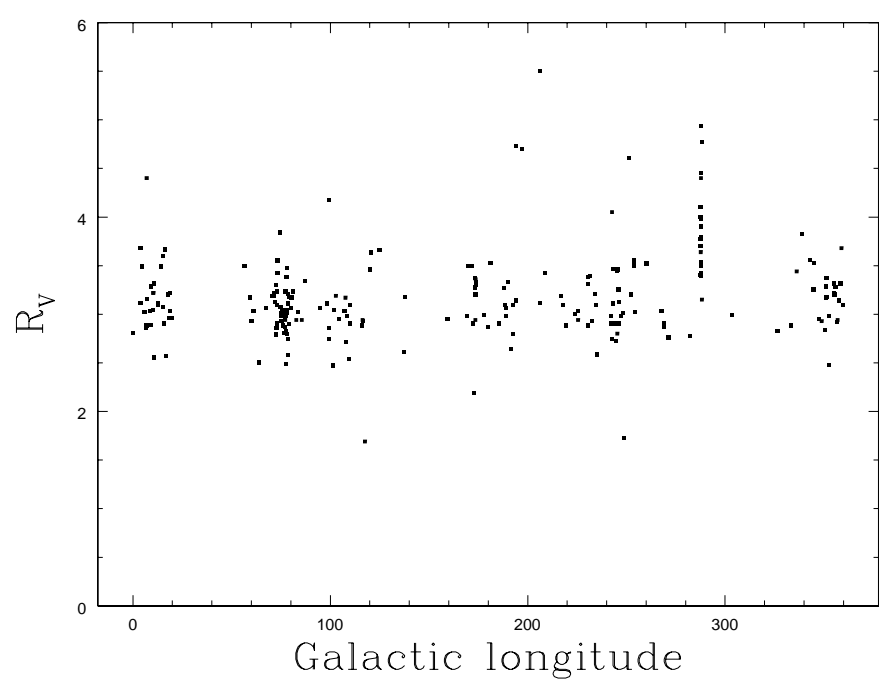

Fig. 8. $R_{V}$ vs. galactic longitude for the total sample. Longitudes of Cygnus and Carina are 75 and 287, respectively.

In Figs. 7 and 8 we show $E(B-V)$ and $R_{V}$ vs. the galactic longitude, respectively. A significant point concerns the behaviour of the lines of sight towards Cygnus in comparison with those towards Carina. The $R_{V}$ values towards Cygnus average 3.1, while those towards Carina average 4.0 (see Fig. 9). On the other hand $E(B-V)$ of Cygnus spread between 0.3 and 2, while those in Carina are concentrated around 0.5. That means that Carina stars either exhibit local cloud effects, in spite of their relatively large distance, or that the general interstellar medium towards Carina has a higher $R_{V}$ than that typical for most of the Galaxy. We favour the first possibility

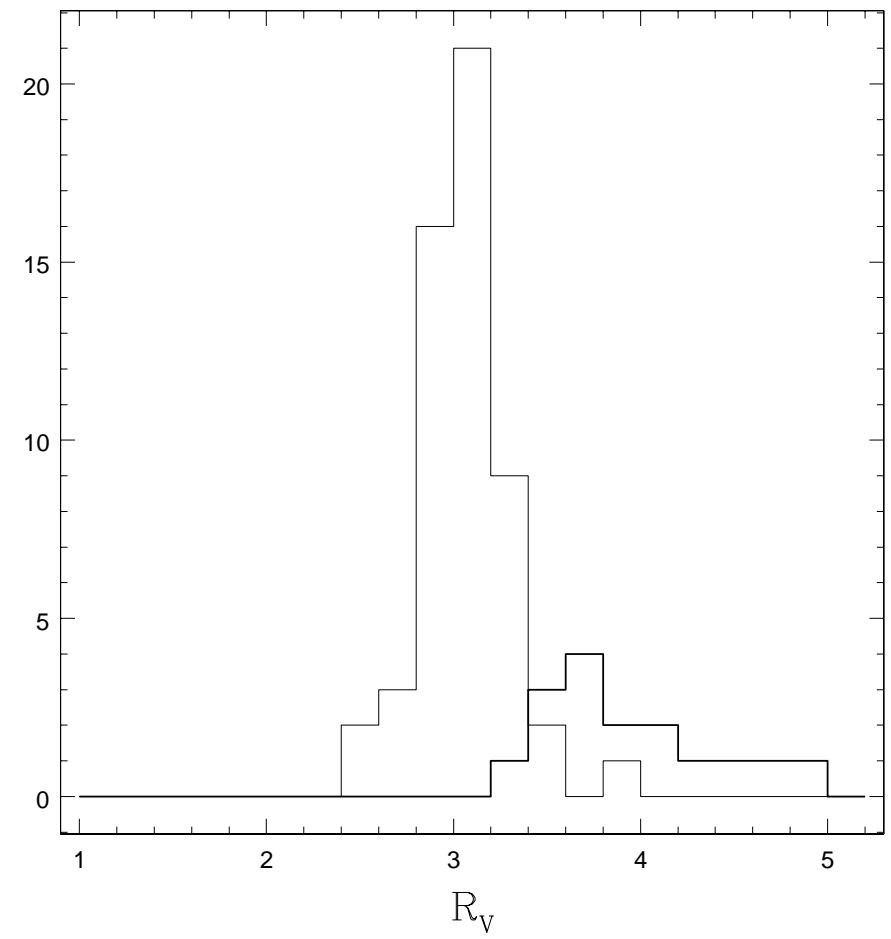

Fig. 9. Histogram of the stars (complet sample) belonging to Cygnus (thin line) and Carina (thick line).

because it is consistent with the fact that in the direction of Carina the lines of sight cross a substantial portion of interarm region, where we do not expect a significant presence of interstellar material. We suggest therefore that the anomaly refers to the Carina association.

\section{References}

Cardelli, J. A., Clayton, G. C., \& Mathis, J. S. 1989, ApJ, 345, 245 (CCM 89)

Cutri, R. M., Skrutskie, M. F., Van Dyk, S., et al. 2001, Explanatory Supplement to the 2MASS Second Incremental Data Release http://pegasus . phast . umass . edu/GradProg/2mass . html Fitzpatrick, E. L. 1999, PASP, 111, 63

Garmany, C. D., Conti, P. S., \& Chiosi, C. 1982, ApJ, 263, 777

Neugebauer, G., \& Leighton, R. B. 1969, Two-Micron Sky Survey, NASA SP-3047 (Washington: NASA)

Panagia, N. 1973, AJ, 78, 929

Patriarchi, P., Morbidelli, M., Perinotto, M., \& Barbaro, G. 2001, A\&A, 372, 644 (Paper I)

Rieke, G. H., \& Lebofsky, M. J. 1985, ApJ, 288, 618

Skórzyński, W., Strobel, A., \& Galazutdinov, G. A. 2003, A\&A, 408, 297

Wegner, W. 1994, MNRAS, 270, 229 